\title{
Role of SAMHD1 nuclear localization in restriction of HIV-1 and SIVmac
}

\author{
Alberto Brandariz-Nuñez ${ }^{1 \dagger}$, Jose Carlos Valle-Casuso ${ }^{1 \dagger}$, Tommy E White $^{1}$, Nadine Laguette ${ }^{2}$, Monsef Benkirane ${ }^{2}$, \\ Jurgen Brojatsch ${ }^{1}$ and Felipe Diaz-Griffero ${ }^{1 *}$
}

\begin{abstract}
Background: SAMHD1 is a nuclear protein that blocks lentiviral infection before reverse transcription in macrophages and dendritic cells. The viral accessory protein Vpx overcomes the SAMHD1-mediated lentiviral block by inducing its proteasomal degradation.

Results: Here, we identified the nuclear localization signal (NLS) of SAMHD1, and studied its contribution to restriction of HIV-1 and SIVmac. By studying the cellular distribution of different SAMHD1 variants, we mapped the nuclear localization of SAMHD1 to residues ${ }^{11} \mathrm{KRPR}^{14}$. Mutagenesis of these residues changed the cellular distribution of SAMHD1 from the nucleus to the cytoplasm. SAMHD1 mutants that lost nuclear localization restricted HIV-1 and SIV as potently as the wild type protein. Interestingly, SAMHD1 mutants that localized to the cytoplasm were not degraded by nuclear Vpx alleles. Therefore, nuclear Vpx alleles require nuclear localization of SAMHD1 in order to induce its degradation. In agreement, SIVmac viruses encoding Vpx did not overcome the restriction imposed by the cytoplasmic variants of SAMHD1.

Conclusions: We mapped the NLS of SAMHD1 to residues ${ }^{11} \mathrm{KRPR}^{14}$ and studied the contribution of SAMHD1 nuclear localization to restriction of HIV-1 and SIV. These experiments demonstrate that cytoplasmic variants of SAMHD1 potently block lentiviral infection and are resistant to Vpx-mediated degradation. The nuclear Vpx alleles studied here are only capable of degrading a nuclearly localized SAMHD1 suggesting that Vpx-mediated degradation of SAMHD1 is initiated in the nucleus.
\end{abstract}

Keywords: SAMHD, Restriction, Nuclear localization, Vpx, HIV-1, SIV, Degradation

\section{Background}

Infection of primary macrophages and dendritic cells by Simian Immunodeficiency virus (SIV) requires the accessory protein Vpx, which is encoded in the SIV genome [1]. SIV particles without Vpx (SIV $\Delta V \mathrm{px}$ ) are unable to infect primary macrophages. $\mathrm{Vpx}$ is essential for the ability of SIV to infect primary macrophages in vitro and for viral dissemination and pathogenesis in vivo [2-5]. Vpx is incorporated into viral particles suggesting that it might be acting immediately after viral fusion with target

\footnotetext{
* Correspondence: felipe.diaz-griffero@einstein.yu.edu

${ }^{\dagger}$ Equal contributors

'Department of Microbiology and Immunology, Albert Einstein College of Medicine Bronx, Bronx, NY 10461, USA

Full list of author information is available at the end of the article
}

cells [6-9]. Viral reverse transcription is prevented in primary macrophages when cells are infected with Vpx-deficient SIV or HIV-2 [10-14]. These experiments suggest that when Vpx is incorporated into viral particles, the virus overcomes a pre-reverse transcription block in macrophages and dendritic cells. Remarkably, Vpx also increases the ability of HIV-1 to infect macrophages and dendritic cells when Vpx is incorporated into HIV-1 particles or supplied in trans $[15,16]$. This suggests that the block imposed by macrophages to SIV $\Delta \mathrm{Vpx}$ is similar to the one imposed by macrophages to HIV-1.

Recent work identified SAMHD1 as the protein that blocks infection of SIV $\Delta \mathrm{Vpx}$ and HIV-1 before reverse transcription in macrophages and dendritic cells $[17,18]$. Mutations in SAMHD1 cause Aicardi-Goutières syndrome, which is a genetic disease that causes encephalopathy closely resembling sequelae from congenital infection

\section{Biomed Central}


[19]. SAMHD1 contains a sterile alpha motif (SAM) and a histidine-aspartic (HD) domain. SAM domains are protein interaction modules that mediate interaction with other SAM domains or non-SAM domain-containing proteins [19]. Additionally, SAM domains in other proteins bind a specific sequence of DNA acting as transcription activators or repressors [20]. The HD domain is found in a super family of enzymes with a predicted phosphohydrolase activity [21]. In agreement, recent work has demonstrated that SAMHD1 is a dGTP-regulated deoxynucleotide triphosphohydrolase that might be involved in decreasing the overall cellular levels of triphosphodeoxynucleotides [22-24].

SAMHD1 localizes to the nucleus in human fibroblasts as demonstrated by subcellular localization studies either using antibodies against the endogenous protein or the study of a GFP-SAMHD1 fusion construct [19]. Mechanistic studies have suggested that $\mathrm{Vpx}$ induces the proteasomal degradation of SAMHD1[17,18]. In addition, localization studies of the Vpx protein during SIV or HIV-1 infection correlated the nuclear localization of Vpx with its ability to promote productive infection in primary macrophages $[5,15,25,26]$, suggesting that Vpx might overcome the viral-block imposed by SAMHD1 in the nucleus. Therefore, we decided to study the role of SAMHD1 nuclear localization in its ability to block HIV1 and SIVmac infection. For this purpose, we first identified the NLS of SAMHD1 and analyzed the ability of SAMHD1 NLS variants to block infection. In addition, we tested the ability of Vpx to induce the degradation of SAMHD1 NLS variants.

\section{Results \\ SAMHD1 is a nuclear protein}

Endogenous SAMHD1 localizes to the nuclear compartment of human MRC-5 fibroblasts [19]. Here, we tested the localization of endogenous SAMHD1 in monocytederived macrophages (MDM), PMA-differentiated THP-1 cells, and HeLa cells. In addition, we studied the localization of epitope tagged exogenous SAMHD1 in HeLa cells. As previously reported, in all cell types studied here, endogenously and exogenously expressed SAMHD1 localized to the nuclear compartment Additional file (1A-C). Interestingly, SAMHD1 did not co-localized with fibrilarin suggesting that SAMHD1 is in the nucleoplasm, but not in the nucleolus Additional file (1D). Overall these results indicate that SAMHD1 localizes to the nucleus, and that the cytoplasm contains a very small fraction of the total amount of SAMHD1 in the cell. To understand the contribution of SAMHD1 nuclear localization to restriction of HIV-1 and SIVmac, we first investigated the region of SAMHD1 that contributes to its nuclear import.

\section{The nuclear localization signal (NLS) of SAMHD1 is contained in the first 150 amino acids}

Protein transport from the cytosol to the nucleus generally requires the existence of a NLS [27]. To test for the presence of an NLS in SAMHD1, we fused the green fluorescent protein (GFP) to different regions of the SAMHD1 protein (Figure 1A) and tested the different constructs for nuclear localization and expression in HeLa cells (Figure 1B-C).

Our mapping studies revealed that SAMHD1 contains a putative NLS in the first 150 amino acids (Figure 1). The GFP fusion with the full-length SAMHD1 [GFPSAMHD1(1-626)] localized to the nucleus similar to the endogenously expressed protein (Figure 1B). In contrast to the fusion GFP-SAMHD1(329-626) that contains the C-terminal half of the protein, GFP-SAMHD1(1-328) exclusively localized to the nucleus (Figure 1B), suggesting that the putative NLS is located in the first 328 amino acids. To further narrow the location of a potential NLS, we tested GFP-SAMHD1(1-150) and GFP-SAMHD1 (151-328). Contrary to GFP-SAMHD1(151-328), the GFP fusion construct that contains the first 150 amino acids of SAMHD1 exclusively localized to the nucleus [GFPSAMHD1(1-150)]. These results suggested that the sequence required for nuclear localization of SAMHD1 is comprised within the first 150 amino acids, which is in agreement with the nuclear localization of a naturally occurring truncation of SAMHD1 that only contains the first 149 amino acids [19].

\section{Site-directed mutagenesis of the putative SAMHD1 NLS}

Analysis of the first 150 amino acids of SAMHD1 revealed that the peptide ${ }^{11} \mathrm{KRPR}^{14}$ starting at position 11 is a potential NLS (Figure 2A) [28]. To test this hypothesis, we mutagenized the potential NLS in the GFP-SAMHD1(1-626) construct and tested these mutants for subcellular localization and expression (Figure 2B-C). All tested GFPSAMHD1(1-626) mutants of the ${ }^{11} \mathrm{KRPR}^{14}$ peptide localized to the cytoplasmic compartment (Figure 2B). Thus, the ${ }^{11} \mathrm{KRPR}^{14}$ sequence may act as nuclear localization signal of SAMHD1. We ruled out the potential influence of the GFP in the localization of SAMHD1 by performing similar changes in a FLAG-tagged SAMHD1 construct. We tested the subcellular localization and expression of the FLAGtagged SAMHD1 variants (Figure 2D-E). Similarly, we found that changes in the NLS localized SAMHD1 to the cytoplasm. To confirm these results, we also tested the localization of a deletion construct of SAMHD1 that does not contain the first 14 amino acids. In agreement with our findings that the initial 14 amino acids of SAMHD1 contain the NLS, the construct SAMHD1(15-626)-FLAG localized exclusively to the cytoplasm Additional file (2). Next, we tested whether this NLS is sufficient to target unrelated proteins to the nucleus. 
The nuclear localization signal of SAMHD1 is transferable To test whether the NLS of SAMHD1 is transferable, we incorporated the NLS of SAMHD1, ${ }^{11} \mathrm{KRPR}^{14}$, to the $\mathrm{N}$ terminus of a GFP protein fused to the viral protein muNS (GFP-muNS) from avian reovirus that localizes to the cytoplasm (Figure 3A) [29,30]. Remarkably, the construct expressing KRPR-GFP-muNS was exclusively localized to the nuclear compartment (Figure 3A, C). This suggests that the SAMHD1 NLS is sufficient to change the localization of cytosolic GFP-muNS. Similar results were obtained by fusing the NLS of SAMHD1 to a wild type GFP protein (Figure 3B, C). Overall these results demonstrated that the NLS of SAMHD1, ${ }^{11} \mathrm{KRPR}^{14}$, is sufficient to allow the import of an unrelated protein to the nucleus. Thus, the ${ }^{11} \mathrm{KRPR}^{14} \mathrm{se}$ quence is the nuclear localization signal of SAMHD1.

\section{Contribution of SAMHD1 nuclear localization to restriction of HIV-1}

The nuclear localized SAMHD1 blocks retroviral infection before reverse transcription [10-14]. Here, we wanted to test whether nuclear localization of SAMHD1 is required for restriction of HIV-1. To test for this requirement, we challenged human monocytic U937 cells stably transduced with wild type and mutant SAMHD1 proteins with increasing amounts of HIV-1 (Figure 4A, B). The SAMHD1 NLS mutants that localized to the cytoplasm restricted HIV-1 as efficiently as the wild type protein (Figure 4B, C). Similar HIV-1 restriction was observed when expressing the different SAMHD1 variants in THP-1 cells that were stably silenced for expression of endogenous SAMHD1 (Figure 4D, E). Altogether these results indicated that restriction of HIV-1 by SAMHD1 does not require the presence of SAMHD1 in the nucleus.

\section{Vpx-mediated degradation of SAMHD1 requires nuclear} localization of SAMHD1

The SIV accessory protein Vpx overcomes SAMHD1 restriction by triggering its degradation $[17,18]$. As shown, SAMHD1 is localized to the nuclear compartment when endogenously or exogenously expressed [19]. In agreement, localization studies of the $\mathrm{Vpx}$ protein

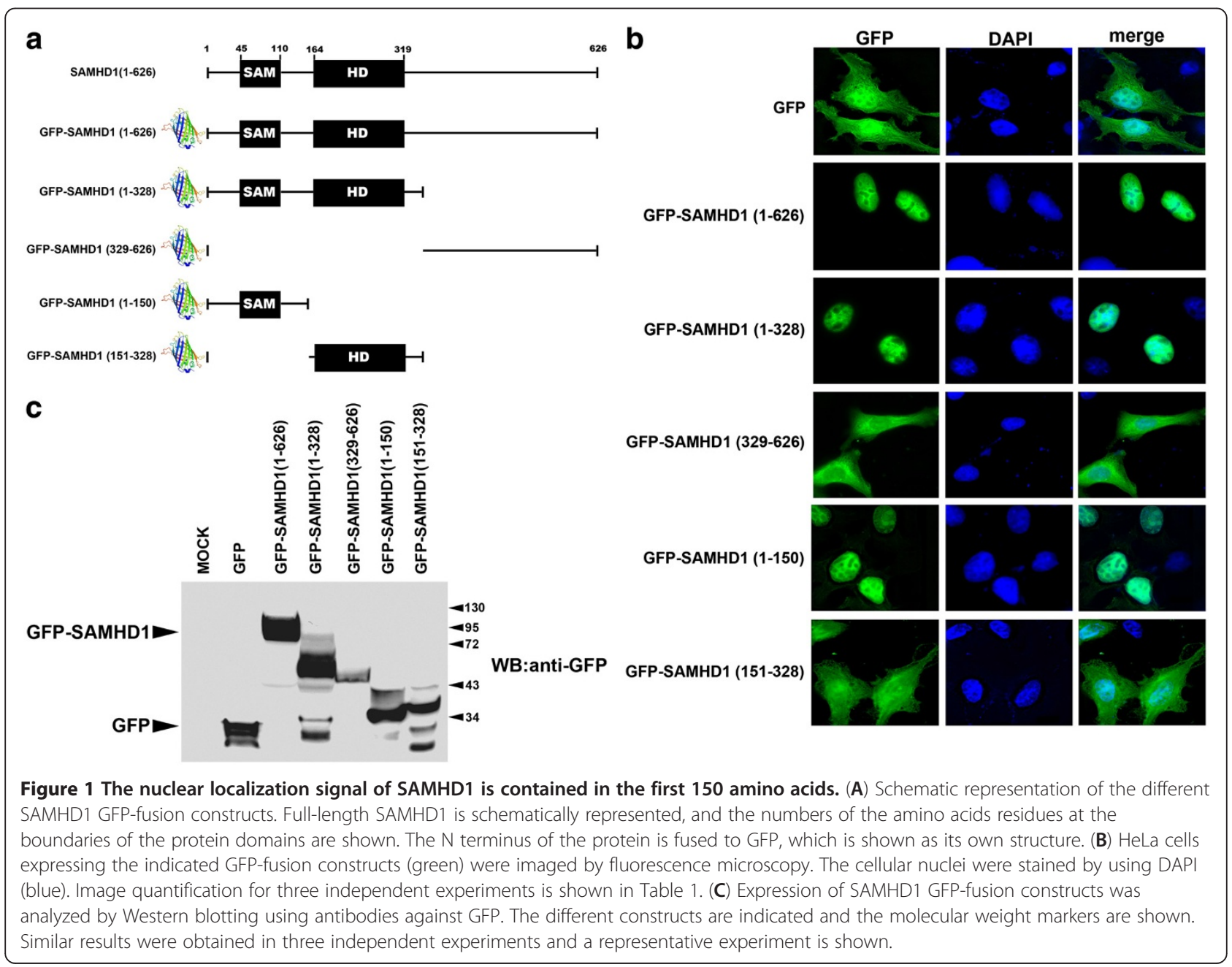


Table. 1 Immunostaining data

\begin{tabular}{|c|c|c|c|c|c|c|c|c|c|c|}
\hline \multirow[t]{2}{*}{ SAMHD1 } & \multicolumn{3}{|c|}{ Experiment 1} & \multicolumn{3}{|c|}{ Experiment 2} & \multicolumn{3}{|c|}{ Experiment 3} & \multirow[t]{2}{*}{ Figure } \\
\hline & $\begin{array}{l}\text { Exclusively } \\
\text { nuclear }\end{array}$ & $\begin{array}{l}\text { Exclusively } \\
\text { cytoplasm }\end{array}$ & $\begin{array}{c}\text { Throughout } \\
\text { cell }\end{array}$ & $\begin{array}{c}\text { Exclusively } \\
\text { nuclear }\end{array}$ & $\begin{array}{l}\text { Exclusively } \\
\text { cytoplasm }\end{array}$ & $\begin{array}{c}\text { Throughout } \\
\text { cell }\end{array}$ & $\begin{array}{l}\text { Exclusively } \\
\text { nuclear }\end{array}$ & $\begin{array}{l}\text { Exclusively } \\
\text { cytoplasm }\end{array}$ & $\begin{array}{c}\text { Throughout } \\
\text { cell }\end{array}$ & \\
\hline GFP-SAMHD1 & 184 & 0 & 16 & 190 & 0 & 10 & 188 & 0 & 12 & 1B \\
\hline GFP-SAMHD1 (1-328) & 176 & 0 & 24 & 180 & 0 & 20 & 177 & 0 & 23 & $1 \mathrm{~B}$ \\
\hline GFP-SAMHD1(329-626 & 0 & 40 & 160 & 0 & 46 & 154 & 0 & 38 & 162 & 1B \\
\hline GFP-SAMHD1(1-150) & 156 & 0 & 44 & 160 & 0 & 40 & 164 & 0 & 36 & $1 \mathrm{~B}$ \\
\hline GFP-SAMHD1(151-328) & 0 & 0 & 200 & 0 & 0 & 200 & 0 & 0 & 200 & 1B \\
\hline GFP-SAMHD1 K11A & 0 & 188 & 12 & 0 & 190 & 10 & 0 & 192 & 8 & $2 \mathrm{~B}$ \\
\hline GFP-SAMHD1 R12A & 0 & 190 & 10 & 0 & 194 & 6 & 0 & 188 & 12 & $2 \mathrm{~B}$ \\
\hline GFP-SAMHD1 R14A & 0 & 192 & 8 & 0 & 196 & 4 & 0 & 186 & 14 & $2 \mathrm{~B}$ \\
\hline SAMHD1-FLAG & 196 & 0 & 4 & 194 & 0 & 6 & 196 & 0 & 4 & $2 \mathrm{D}$ \\
\hline SAMHD1-FLAG K11A & 0 & 194 & 6 & 0 & 192 & 8 & 196 & 0 & 4 & $2 \mathrm{D}$ \\
\hline SAMHD1-FLAG R12A & 0 & 196 & 4 & 0 & 194 & 6 & 0 & 192 & 4 & $2 \mathrm{D}$ \\
\hline SAMHD1-FLAG R14A & 0 & 192 & 8 & 0 & 190 & 10 & 0 & 188 & 12 & $2 \mathrm{D}$ \\
\hline GFP-muNS & 0 & 200 & 0 & 0 & 200 & 0 & 0 & 200 & 0 & $3 \mathrm{~A}$ \\
\hline KRPR-GFP-muNS & 150 & 0 & 50 & 146 & 0 & 54 & 152 & 0 & 48 & $3 \mathrm{~A}$ \\
\hline GFP & 0 & 0 & 200 & 0 & 0 & 200 & 0 & 0 & 200 & $3 B$ \\
\hline KRPR-GFP & 140 & 0 & 60 & 146 & 0 & 54 & 142 & 0 & 58 & $3 \mathrm{~B}$ \\
\hline SAMHD1-FLAG (U937) & 194 & 0 & 6 & 190 & 0 & 10 & 196 & 0 & 4 & $4 C$ \\
\hline SAMHD1-FLAG K11A (U937) & 194 & 0 & 6 & 190 & 0 & 10 & 196 & 0 & 4 & $4 C$ \\
\hline SAMHD1-FLAG R12A (U937) & 0 & 192 & 8 & 0 & 196 & 4 & 0 & 188 & 14 & $4 C$ \\
\hline SAMHD1-FLAG R14A (u937) & 0 & 190 & 10 & 0 & 184 & 16 & 0 & 186 & 14 & $4 C$ \\
\hline SAMHD1 (MDM) & 196 & 0 & 4 & 192 & 0 & 8 & 198 & 0 & 2 & AF1A \\
\hline SAMHD1 (THP1 + PMA) & 198 & 0 & 2 & 196 & 0 & 4 & 200 & 0 & 0 & AF1A \\
\hline SAMHD1 (Endogenous in Hela) & 194 & 0 & 6 & 200 & 0 & 0 & 196 & 0 & 4 & AF1A \\
\hline SAMHD1 (Tramsient expression) & 198 & 0 & 2 & 200 & 0 & 0 & 194 & 0 & 6 & AF1B \\
\hline SAMHD1 (Stable expression & 196 & 0 & 4 & 196 & 0 & 4 & 200 & 0 & 0 & AF1B \\
\hline SAMHD1-FLAG (15-626) & 0 & 194 & 6 & 0 & 196 & 4 & 0 & 190 & 10 & AF2A \\
\hline
\end{tabular}

Three independent experiments were performed. Cells were scored visually for nuclear and cytoplasmic distribution. In every experiment two hundred cells were counted. The analysis of the distribution was performed in human Hela cells or otherwise indicated. MDM = Monocyte derived macrophages. PMA = phorbol-12-myristate-13-acetate. 


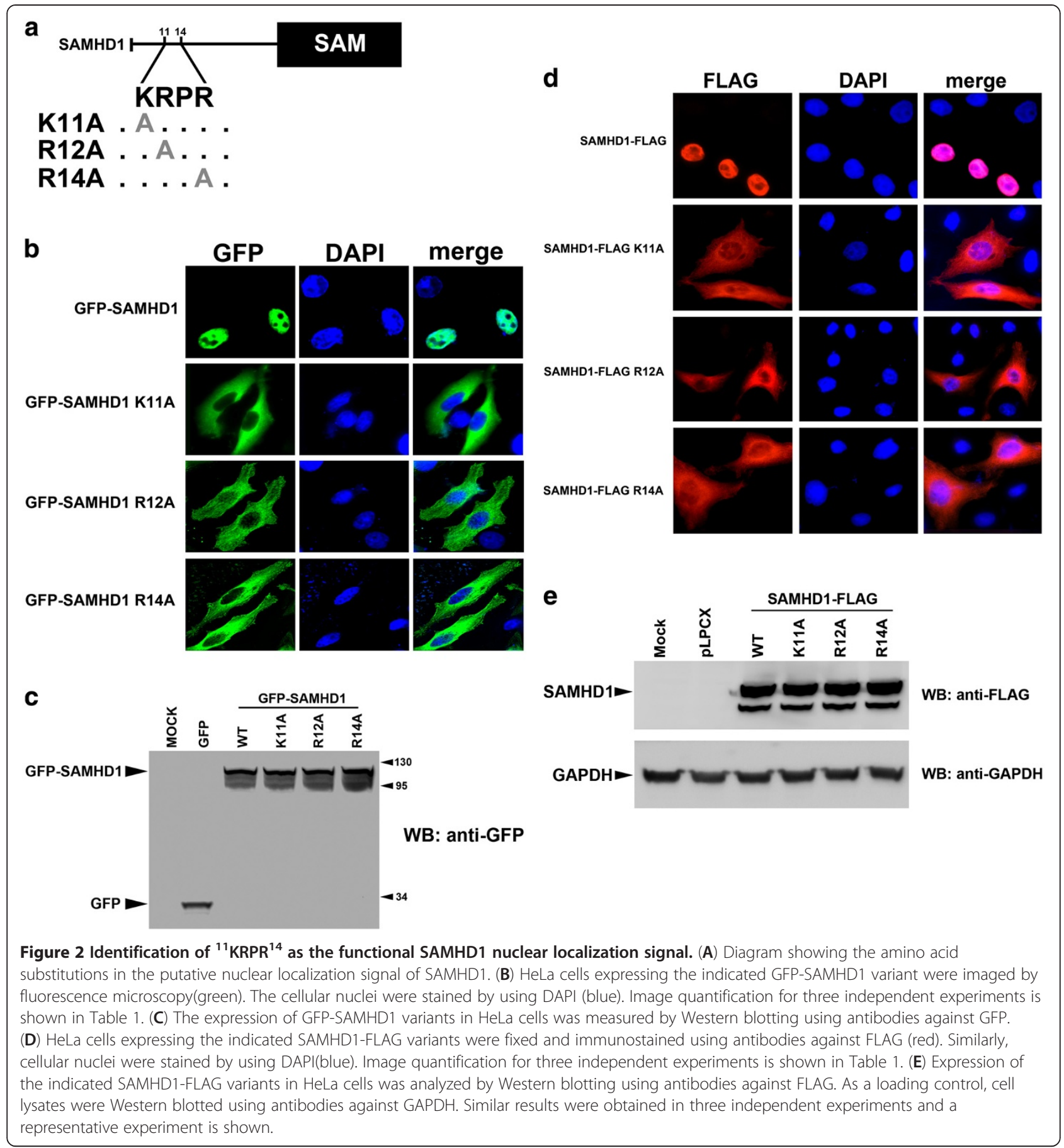

during lentiviral infection correlated the nuclear localization of $\mathrm{Vpx}$ with its ability to promote productive infection in primary macrophages [5,15,25,26], which suggests that $\mathrm{Vpx}$ might overcome the viral-block imposed by SAMHD1 in the nucleus. We hypothesized that nuclear localization of SAMHD1 is required for the ability of Vpx to induce its degradation and overcome restriction. For this purpose, we measured the ability of nuclear and cytoplasmic Vpx proteins to induce degradation of SAMHD1 variants that localized to the cytoplasm (Figure 5A). In agreement with our hypothesis, the nuclear alleles of $\mathrm{Vpx}$ : Vpx from the ROD strain of HIV-2 $\left(\mathrm{Vpx}_{\mathrm{ROD}}\right)$ and $\mathrm{Vpx}$ from $\mathrm{SIVmac}_{251}$ $\left(\mathrm{Vpx}-\mathrm{mac}_{251}\right)$ Additional file (3) [31], did not trigger the degradation of the cytoplasmic variant SAMHD1-K11A (Figure 5A). As expected, $\mathrm{Vpx}_{\mathrm{ROD}}$ and $\mathrm{Vpx}-\mathrm{mac}_{251}$ 


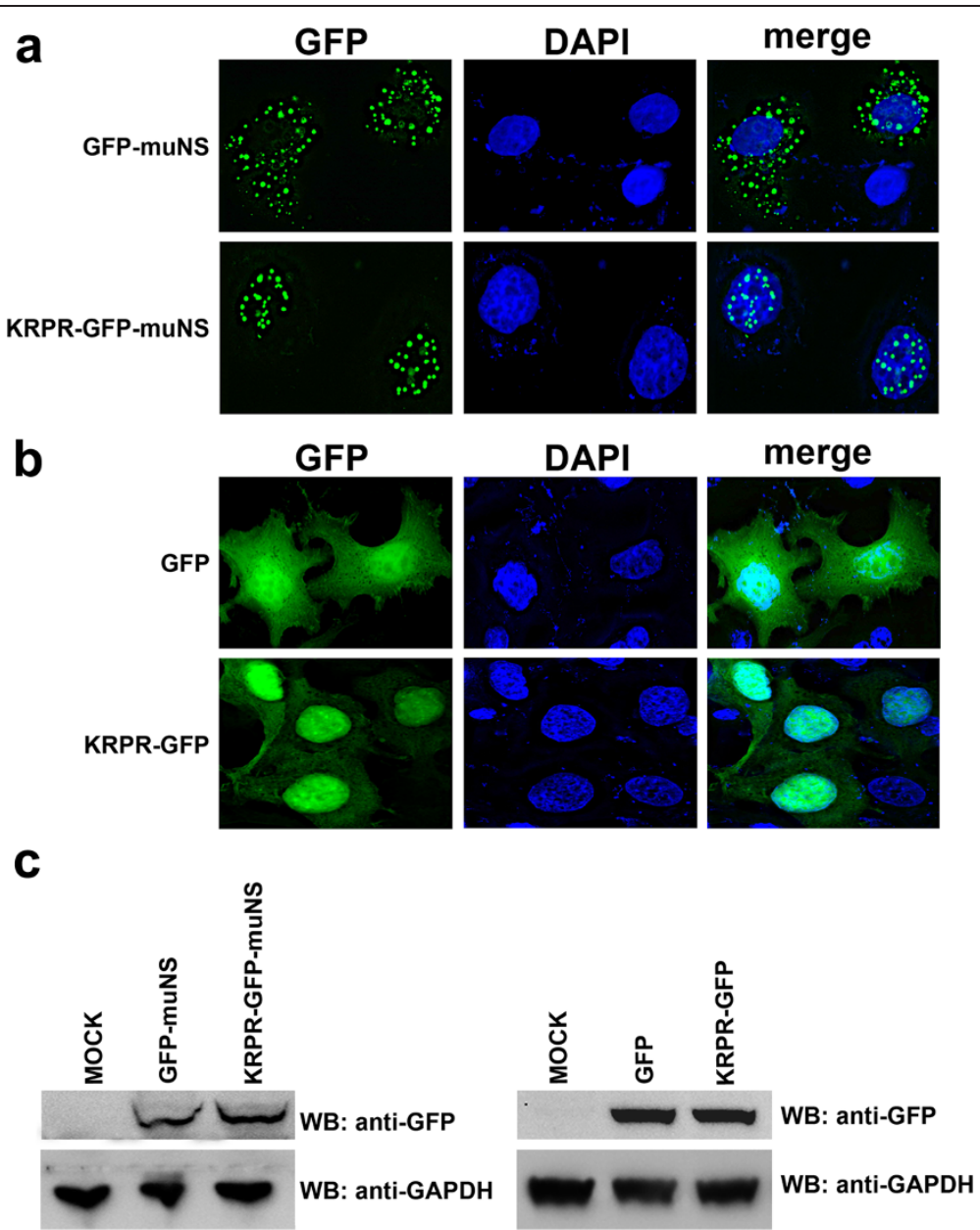

Figure 3 The nuclear localization signal of SAMHD1 is transferable. (A) HeLa cells expressing a fusion between the NLS of SAMHD1 and the cytoplasmic GFP-muNS protein (KRPR-GFP-muNS) were imaged by fluorescence microscopy (green). As a control, the cytoplasmic distribution of the GFP-muNS protein is shown. Cellular nuclei were stained by using DAPI (blue). Image quantification for three independent experiments is shown in Table 1. (B) Similarly, HeLa cells expressing a fusion between the NLS of SAMHD1 and GFP (KRPR-GFP) were imaged by fluorescence microscopy(green). Cellular nuclei were stained by using DAPI (blue). Image quantification for three independent experiments is shown in Table 1. (C) The expression of the indicated fusion proteins was analyzed by Western blotting using antibodies against GFP. As loading controls, cell lysates were Western blotted using antibodies against GAPDH. Similar results were obtained in three independent experiments and a representative experiment is shown.

potently degraded the nuclearly localized wild type SAMHD1 [31]. Remarkably, a cytoplasmic allele of Vpx, $\mathrm{Vpx}$ from HIV-2B $\left(\mathrm{Vpx}_{2 \mathrm{~B}}\right)$ Additional file (3) [31], induced degradation of the cytoplasmic SAMHD1-K11A (Figure 5A). As controls, we used the Vpx protein from $\mathrm{SIV}_{\mathrm{Rcm}-\mathrm{ng}}\left(\mathrm{Vpx}_{\mathrm{Rcm}-\mathrm{ng}}\right)$ that is unable to induce degradation of mutant and wild type SAMHD1 [31]. Next, we tested the ability of SIVmac to overcome the restriction imposed by SAMHD1 (Figure 5B). In agreement with our results that $\mathrm{Vpx}$ from $\mathrm{SIVmac}$ is unable to trigger the degradation of SAMHD1-K11A, we found that SIVmac is not capable of overcoming the restriction imposed by SAMHD1-K11A (Figure 5B). As expected, SIVmac $\Delta \mathrm{Vpx}$ did not overcome the restriction imposed by any of the tested SAMHD1 variants (Figure 5B). These experiments suggested that Vpx nuclear alleles require nuclearly localized SAMHD1 in order to induce its degradation and to overcome restriction.

The observation that the different cytoplasmic variants of SAMHD1 described in this work blocked HIV-1 infection suggested that restriction does not require nuclear localization of SAMHD1. Therefore, we tested whether SAMHD1 changes localization upon HIV-1-GFP infection. For this purpose we infected PMA-treated U937 cells stably expressing SAMHD1 or SAMHD1-K11A with HIV-1. At different hours post-infection, samples were fixed, and the localization of SAMHD1 was determined Additional 


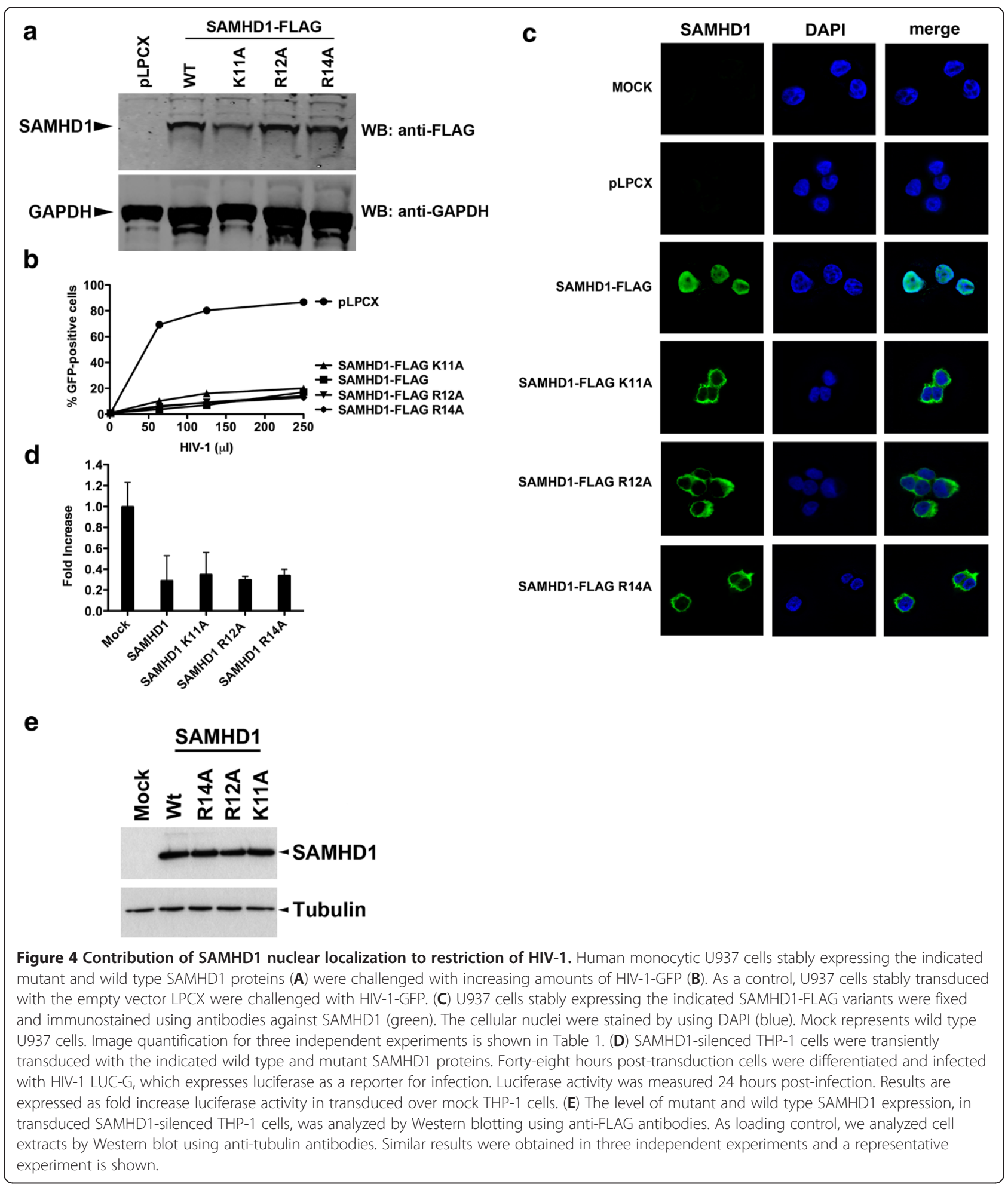

file (4). Even though PMA-treated U937 cells stably expressing SAMHD1 or SAMHD1-K11A potently restricted HIV-1 infection Additional file (4), we did not observe changes in the subcellular localization of SAMHD1.

\section{Discussion}

Here, we mapped the nuclear localization signal of the restriction factor SAMHD1 and evaluated the impact of SAMHD1 cellular distribution on restriction of HIV-1 and SIVmac. Remarkably, mutations in the NLS of 

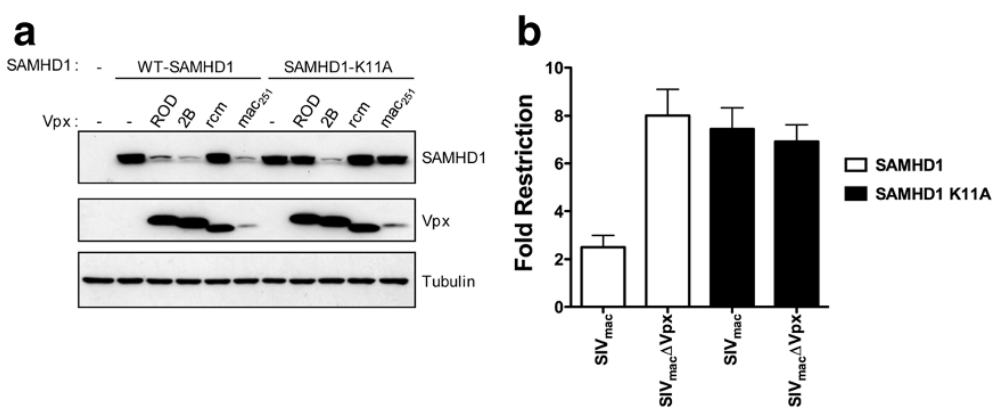

Figure $\mathbf{5} \mathrm{Vpx}_{\mathrm{n}}$-induced degradation of SAMHD1 requires nuclear localization of SAMHD1. (A) HeLa cells were cotransfected with plasmids

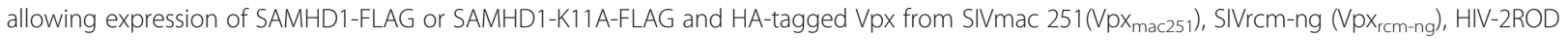
$\left(V p x_{R O D}\right)$ or $\mathrm{HIV}-2 B\left(V \mathrm{Px}_{2 \mathrm{~B}}\right)$. Thirty-six hours post-transfection the cells were harvested, and the expression levels of SAMHD1 and Vpx were analyzed by Western blot using anti-FLAG and HA antibodies. As a loading control, cell extracts were Western blotted using antibodies against Tubulin. Similar results were obtained in three independent experiments and a representative experiment is shown. (B) Human monocytic U937 cells stably expressing the indicated mutant or wild type SAMHD1 proteins were challenged with SIVmac or SIVmac $\Delta V$ px -GFP reporter viruses Infection was determined by measuring the percentage of GFP-positive cells. Fold of restriction was calculated by dividing the $\%$ of GFP-positive cells resulting from infecting U937 cells containing the empty vector pLPCX by the \% GFP-positive cells resulting from infecting U937 stably expressing the indicated SAMHD1 variant. Similar results were obtained in three independent experiments and standard deviation is shown.

SAMHD1 that caused localization of the protein to the cytosol did not affect the ability of SAMHD1 to block HIV-1 or SIV infection. These results imply that SAMHD1 localization to the cytoplasm is sufficient to restrict HIV-1 infection. Because wild type SAMHD1 is mainly localized to the nucleus [19], taken together these observations suggested that SAMHD1 can deplete the overall cellular pool of deoxynucleoside triphosphates when in nucleus or in the cytosol [22-24]. An alternative possibility is that SAMHD1 is recruited to the cytoplasm in order to deplete the cellular pool of deoxynucleoside triphosphates; however, we did not observe changes on the cellular localization of SAMHD1 when cells were challenged by HIV-1 Additional file (4).

While revising this manuscript, mutations on SAMHD1 (I201N and M254V) that partially localized to the cytosol were described, suggesting that these residues might be contributing to the nuclear localization of SAMHD1[32]. However, due to the limited available data on mutations I201N and M254V, it is difficult to conclude whether these residues have a contribution to the nuclear import of SAMHD1. Current issues include: 1) the absence of functional data for mutants I201N and M254V other than subcellular localization [32], 2) residues I201 and M254 are not surface-exposed in the recently described structure of the HD domain[24], and 3) absence of evidence to demonstrate that these residues are part of a transferable NLS [32]. Future experiments will attempt to test whether I201 and M254 residues contribute to the nuclear localization of SAMHD1.

Wild type SAMHD1 localizes to the nucleus. In agreement, localization studies of the Vpx protein during SIV infection correlated the nuclear localization of Vpx with its ability to promote productive infection in primary macrophages [5,15,25,26]. Overall, this suggests that $\mathrm{Vpx}$ encounters SAMHD1 in the nucleus; therefore, Vpx modulation of SAMHD1 levels might start in the nuclear compartment. To test this hypothesis, we assayed the ability of nuclear localized Vpx proteins $\left(\mathrm{Vpx}_{\mathrm{n}}\right)$ to degrade SAMHD1 when in the nucleus or cytosol. Remarkably, $\mathrm{Vpx}_{\mathrm{n}}$ only degraded nuclear SAMHD1. By contrast, SAMHD1 variants that localized to the cytoplasm were not affected by $\mathrm{Vpx}_{\mathrm{n}}$. These results suggested that $\mathrm{Vpx}_{\mathrm{n}^{-}}$ induced degradation of SAMHD1 begins when both proteins are in the nucleus. In agreement, SIV encoding $\mathrm{Vpx}_{\mathrm{n}}$ was unable to overcome the block imposed by cytoplasmic variants of SAMHD1. These results contribute to our understanding of the mechanism used by $\mathrm{Vpx}_{\mathrm{n}}$ to overcome the lentiviral block imposed by SAMHD1.

The fact that $\mathrm{Vpx}_{\mathrm{n}}$ degrades only nuclear localized SAMHD1 suggests that this encounter is the first step of the degradation process of SAMHD1. The second step might be the export of SAMHD1 to the cytoplasm, as suggested by experiments in which leptomycin $\mathrm{B}$ blocked the $\mathrm{Vpx}_{\mathrm{n}}$-induced degradation of SAMHD1 [33]. The final step is likely to be the cytoplasmic proteasomal degradation of SAMHD1 [10,11,15,34].

SAMHD1 restriction of lentiviruses might occur by a direct or an indirect mechanism. A direct mechanism would imply that SAMHD1 recognizes a specific element in the restricted virus; this further suggests the existence of a viral determinant for restriction. Elegant cross-packaging experiments using HIV and SIV demonstrated that dendritic cells and macrophages are more susceptible to an HIV-1 reporter RNA packaged by an HIV-1 Gag-Pol when compared to an SIV reporter RNA packaged with HIV-1[35]. These experiments suggest that the viral determinant for the block observed in 
dendritic cells and macrophages is the viral RNA. This is in agreement with the idea that SAMHD1 blocks retroviral infection before reverse transcription. Future experiments will attempt to identify whether the block observed by cross-packaging experiments in dendritic cells and macrophages is related to SAMHD1.

An indirect mechanism would imply that the enzymatic activity of SAMHD1 is sufficient to restrict HIV-1 replication. Recent work has demonstrated that the triphosphohydrolase activity of the HD domain of SAMHD1 is involved in decreasing the overall cellular levels of deoxynucleoside triphosphates and causing the restriction [22-24]. Depletion of deoxynucleoside triphosphates might occur either in the vicinity or far from where the virus is undergoing reverse transcription.

\section{Conclusions}

We found that the nuclear localization signal of SAMHD1 mapped to residues ${ }^{11} \mathrm{KRPR}^{14}$. The nuclear localization of SAMHD1 is important for the ability of Vpx to trigger degradation of SAMHD1. This work showed that cytoplasmic variants of SAMHD1 potently block HIV-1 and SIV infection and are resistant to $\mathrm{Vpx}_{\mathrm{n}}$. The $\mathrm{Vpx}_{\mathrm{n}}$ alleles studied here are only capable of degrading a nuclearly localized SAMHD1 suggesting that Vpx-mediated degradation of SAMHD1 is initiated in the nucleus.

\section{Methods \\ Plasmids}

The pEGFP-C1-M3 vector, which expresses full-length avian reovirus muNS non-structural protein, was a generous gift from Dr. Jose Martínez-Costas (University of Santiago de Compostela), and has been described previously [30]. The plasmids pLPCX-SAMHD1 and pLPCXSAMHD1-FLAG expressing SAMHD1 and SAMHD1 fused to a FLAG epitope, respectively, were codon optimized by GENEWIZ. For the expression of fusions with green fluorescent protein (GFP), SAMHD1 sequences were amplified by PCR, digested with appropriate restriction enzymes, and inserted into the pEGFP-C1 vector (Clontech). To generate inserts for the production of pEGFP-C1 recombinant plasmids, we used the following primers: for the production of GFP-SAMHD1 (1-626), the sense primer was 5'-GCGGAATTCTATGCAGAGA GCTGATAGCG-3' and the reverse primer was 5'-GCG GGATCCTCACATAGGGTCGTCC-3'; for the production of GFP-SAMHD1(1-328), the sense primer was $5^{\prime}$ GCGGAATTCTATGCAGAGAGCTGATAGCG $-3{ }^{\prime}$ and the reverse primer was $5^{\prime}$-GCGGGATCCTCAGTTATT CTGAATGCCCAGG -3'; for the production of GFPSAMHD1 (329-626), the sense primer was 5'-GCGGAA TTCTTTCGATTACAAAAGGTTTATC-3' and the reverse primer was 5 '-GCGGGATCCTCACATAGGGT CGTCC-3'; for the production of GFP-SAMHD1 (1-150), the sense primer was $5^{\prime}$ - GCGGAATTCTATGCAGAGA GCTGATAGCG $-3^{\prime}$ and the antisense primer was $5^{\prime}$-GC GGGATCCTCACAGCTGCTTGATATATCTG-3', and for the production of GFP-SAMHD1 (151-328), the sense primer was 5'-GCG GAA TTC T GGC GGA GGC TAC TAT GTC - 3 ' and the reverse primer was 5' - GCGGGA TCCTCAGTTATTCTGAATGCCCAGG-3'. The correct orientation of the inserts was confirmed by sequencing and restriction analysis.

To express the SAMHD1 NLS fused to the $\mathrm{N}$ terminus of GFP-muNS the recombinant plasmids pEGFP-C1-M3 [30] was subjected to PCR amplification with the following primers: the forward primer was 5' GCGGGATCCACCATGGGAAAGAGACCCAGGACC ATGGTGAGCAAGGGCGAG-3, and the reverse primer was 5'-GCGTCTAGATTACAGATCATCCACCAATTC TTC-3'. PCR products were digested and cloned into the BamHI and XbaI sites of pCDNA3.1/Zeo.

To express the SAMHD1 NLS fused to the N terminus of GFP the plasmid pEGFP-C1 (Clontech) was subjected to PCR amplification with the following primers: the forward primer was 5'-GCGGGATCCACCATGGGAAA GAGACCCAGGACCATGGTGAGCAAGGGCGAG-3, and the reverse primer was 5'-GCGTCTAGATTACT TGTACAGCTCGTCCATGCC-3'. PCR products were digested and cloned into the BamHI and XbaI sites of pCDNA3.1/Zeo.

The pLPCX-SAMHD1-FLAG plasmid, the pEGFPSAMHD1 plasmid and a QuikChange site-directed mutagenesis kit (Stratagene) were used according to the manufacturer's specifications to generate recombinant plasmids that expressed SAMHD1 variants K11A, R12A and R14A. The following mutagenic oligonucleotide primers were used: for the production of mutation K11A, the sense primer was 5'-GATAGCGAGCAACCCAGC GCGAGACCCAGGTGCGACG-3' and the antisense primer was 5'-GTCGCACCTGGGTCTCGCGCTGG GTTGCTCGCTATC-3'; for the production of mutation R12A, the sense primer was 5'-GATAGCGAGCAACC CAGCAAGGCACCCAGGTGCGACG-3' and the antisense primer was 5'-CGTCGCACCTGGGTGCCTT GCTGGGTTGCTCGCTATC-3'; and for the production of the mutation R14A, the sense primer was $5^{\prime}$-CCC AGCAAGAGACCCGCGTGCGACGATAGCCCCAG-3' and the reverse primer was 5'-CTGGGGCTATCGTCG CACGCGGG TCTCTTGCTGGG- 3 '.

The SAMHD1(15-626)-FLAG plasmid was created by amplifying the gene from pLPCX-SAMHD1-FLAG with the following primers: forward primer was 5' - GCG GAA TTC ACC ATG TGC GAC GAT AGC CCC AGA ACA $C$ and reverse primer was 5'- GCGC ATC GAT TCA CTT GTC GTC GTC GTC CTT GTA GTC CAT AGG GTC GTC CTT AAA C. PCR products were digested and cloned into the EcoRI and ClaI sites of pLPCX. 


\section{Antibodies}

Monoclonal antibodies against SAMHD1 were from Abnova. Anti-FLAG rabbit polyclonal, anti-GAPDH rabbit polyclonal, anti-rabbit-Cy3, anti-mouse Alexa 594, and anti-rabbit Alexa 488 were obtained from Sigma. Anti-GFP rabbit polyclonal antibodies were purchased from Clontech. Anti-fibrillarin monoclonal antibodies were purchased from Cytoskeleton, Inc.

\section{Immunofluorescence microscopy}

Transfections of cell monolayers were performed using Lipofectamine Plus reagent (Invitrogen), according to the manufacturer's instructions. Transfected cells were incubated at $37{ }^{\circ} \mathrm{C}$ for $24 \mathrm{~h}$, unless otherwise stated. For indirect immunofluorescence microscopy, cell monolayers grown on coverslips were transfected. Monolayers were washed twice with PBS and fixed for 15 min with $4 \%$ paraformaldehyde in PBS. Paraformaldehyde-fixed cells were washed twice with PBS, incubated for 4 min in permeabilizing buffer ( $0.5 \%$ Triton $\mathrm{X}-100$ in PBS), and then blocked using PBS containing $2 \%$ bovine serum albumin for $1 \mathrm{~h}$ at room temperature. Subsequently, cells were incubated for $1 \mathrm{~h}$ at room temperature with primary antibodies in blocking buffer. After three washes with PBS, the cells were incubated for $30 \mathrm{~min}$ with secondary antibodies. Cellular nuclei were labeled with 1 $\mu \mathrm{g} / \mathrm{ml}$ of DAPI $(49,69$-diamidino-2-phenylindole) in PBS. Subsequently, samples were mounted for fluorescence microscopy by using the ProLong Antifade Kit (Molecular Probes, Eugene, OR). Images were obtained with a Zeiss Observer.Z1 microscope using a $63 \mathrm{X}$ objective, and deconvolution was performed using the software AxioVision V4.8.1.0 (Carl Zeiss Imaging Solutions).

\section{Protein analysis}

Cellular proteins were extracted with radioimmunoprecipitation assay (RIPA) buffer, as previously described [36]. Detection of proteins by Western blotting was performed using anti-FLAG (Sigma), anti-GAPDH (Sigma), anti-SAMHD1 (Abnova), anti-GFP (clontech), and antirabbit and anti-mouse antibodies conjugated to Alexa Fluor 680(Li-Cor). Bands were detected by scanning blots with the Li-Cor Odyssey Imaging System using the 700 channel.

\section{Generation of cells stably expressing SAMHD1 variants} Retroviral vectors encoding wild-type SAMHD1, SAMHD1 fused to FLAG epitope or SAMHD1 mutants were created using the pLPCX vector (Clontech). Recombinant viruses were produced in $293 \mathrm{~T}$ cells by cotransfecting the pLPCX plasmids with the pVPack-GP and pVPack-VSV-G packaging plasmids (Stratagene). The pVPack-VSV-G plasmid encodes the vesicular stomatitis virus $G$ envelope glycoprotein, which allows efficient entry into a wide range of vertebrate cells [37]. Transduced HeLa and human monocytic U937 cells were selected in $5 \mu \mathrm{g} / \mathrm{ml}$ and $0.2 \mu \mathrm{g} / \mathrm{ml}$ puromycin, respectively.

\section{Infection with viruses expressing green fluorescent protein (GFP)}

Recombinant HIV-1, $\operatorname{SIV}_{\text {mac }}$ and $\operatorname{SIV}_{\text {mac }} \Delta V$ px expressing GFP were prepared as described [38]. All recombinant viruses were pseudotyped with the VSV-G glycoprotein. For infections, $5 \times 10^{4}$ cells seeded in 24-well plates were incubated at $37^{\circ} \mathrm{C}$ with virus for 24 hours. Cells were washed and returned to culture for 48 hours, and the percentage of GFP-positive cells was determined by flow cytometry (Becton Dickinson). Viral stocks were titrated by serial dilution on $\mathrm{Cf} 2 \mathrm{Th}$ cells to determine the concentration of infectious viruses.

\section{Preparation of monocyte-derived macrophages}

Human monocytes were obtained from normal blood donor buffy coats by two-step gradient centrifugation followed by an additional step using the Monocyte Isolation kit II (Miltenyi Biotec) as previously described [39]. Macrophages were obtained by culturing monocytes for 7 days in RPMI 1640 (Invitrogen) supplemented with $10 \%$ FBS (Sigma) and autologous serum at a density of $1.5 \times 10^{5} / \mathrm{cm}^{2}$.

\section{Infection of SAMHD1-silenced THP-1 cells expressing the different SAMHD1 variants}

THP-1 cells stably expressing an shRNA targeting SAMHD1 and C-terminally FLAG- and HA- tagged shRNA-resistant SAMHD1 have been previously described[17]. SAMHD1 proteins harboring mutations K11A, R12A and R14A were generated on the shRNAresistant SAMHD1 backbone in the previously described MMLV-based retroviral vector (pOZ-puro) [40]. Production of retroviral particles was achieved using the standard phosphate calcium procedure to transfect 293T cells (5 $\mu \mathrm{g}$ pOZ construct, $2.5 \mu \mathrm{g}$ packaging plasmid and $2.5 \mu \mathrm{g}$ A-MLV envelope encoding plasmid). Silenced THP-1 cells were transduced or not with retroviral particles 48 hours prior to a 16 hours differentiation step using phorbol-12-myristate-13-acetate (PMA). Cells were subsequently infected with $30 \mathrm{ng}$ of a VSV-G pseudotyped molecular clone of HIV-1 encoding the luciferase gene as a reporter. Luciferase activity was measured $24 \mathrm{hrs}$ post infection and normalized for protein concentration.

\section{Vpx-induced degradation of SAMHD1}

N-terminally FLAG- and HA-tagged Vpxmac251, VpxROD, VpxRCM-NG [17] and HIV-2B have been previously described [31]. HeLa cells were co-transfected with $0.250 \mu \mathrm{g}$ SAMHD1 or SAMHD1-K11A together or not with $3 \mu \mathrm{g}$ Vpx alleles, using the jetPEI kit (Polyplus). 
Thirty-six hours post-transfection the cells were harvested; whole cell extracts prepared and analyzed by Western blotting using antibodies against FLAG or HA.

\section{Additional files}

\section{Additional file 1: Intracellular distribution of SAMHD1 in differents} types of cells. (A) The intracellular distribution of endogenous SAMHD1 was studied in human monocyte derived macrophages (MDM), PMAtreated human THP-1 monocytic cells, and human HeLa cells. Cells were fixed and immunostained using antibodies against SAMHD1(red), as described in Materials and Methods. The cellular nuclei were stained using DAPI (blue). Image quantification for three independent experiments is shown in Table 1. (B) Localization of exogenously expressed SAMHD1 in HeLa cells. Transfected or transduced cells with plasmids expressing the indicated proteins and immunostained using antibodies against SAMHD1(red) or FLAG(red), as indicated. Similarly, the nuclei were stained with DAPI (blue). Image quantification for three independent experiments is shown in Table 1. (C) Expression of SAMHD1 in the indicated cells was analyzed by Western blotting using antibodies against SAMHD1 or FLAG. As a control, cell lysates were Western blotted using antibodies against GAPDH. (D) HeLa cells transfected with SAMHD1-FLAG were stained using antibodies against FLAG(red) and fibrilarin (green). DAPI was used to label the cellular nuclei. Similar results were obtained in three independent experiments, and a representative experiment is shown

Additional file 2: Intracellular distribution of SAMHD1(15-626)-FLAG in HeLa cells. (A) HeLa cells expressing the indicated SAMHD1 variant were fixed and immunostained using antibodies against FLAG(red), as described in Materials and Methods. The cellular nuclei were stained using DAPI (blue). Image quantification for three independent experiments is shown in Table 1. (B) Expression of the indicated SAMHD1 variant in HeLa cells was analyzed by Western blotting using antibodies against FLAG. As a control, cell lysates were Western blotted using antibodies against GAPDH. Similar results were obtained in three independent experiments and a representative experiment is shown.

Additional file 3: Intracellular distribution of the different Vpx alleles. (A) HeLa cells were co-transfected with $0.25 \mathrm{ug}$ of SAMHD1 or SAMHD1-K11A together or not with $3 \mu \mathrm{g}$ of the indicated HA tagged Vpx allele similar to the degradation experiments performed in Figure 5A. Thirty-six hours post-transfection the cells were fixed and stained using antibodies against SAMHD1 (green) and HA(red). In this experiment SAMHD1 was stained using a suboptimal concentration of antibody that do not stain the endogenous SAMHD1 from HeLa cells. The nuclei were stained with DAPI (blue). (B) Image quantification was performed by counting 200 Vpx-positive cells. Results are expressed as the number of Vpx-positive cells where co-staining of Vpx and SAMHD1 was observed (Co-staining). Similarly, we show the number of Vpx-positive cells where SAMHD1 was not observed (No Co-staining). This experiment was repeated three times and standard deviation is shown.

Additional file 4: SAMHD1 localization does not change upon infection of HIV-1. (A) Human monocytic U937 cells stably expressing the indicated SAMHD1 variants were challenged by HIV-1-GFP virus (green) using an amount of virus that will infect $\sim 40 \%$ of cells containing the empty vector pLPCX. Cells were fixed at the indicated hours postinfection (h.p.i.) and immunostained using antibodies against SAMHD1 (red), as described in Materials and Methods. The nuclei were stained with DAPI (blue). (B) Image quantification for three independent experiments is shown. (C) Similar challenges were incubated for fortyeight hours and infection was determined by measuring the percentage of GFP-positive cells. Similar results were obtained in three independent experiments and the standard deviation is shown.

\section{Competing interests}

The authors declare that they have no competing interests.

\section{Acknowledgements}

We thank Thomas Meier for helpful discussions. We thank Frank Kirchhoff and Jose Martinez-Costas for providing reagents. SIV vector with or without Vpx were generously provided by Paul Bieniasz. This work was funded by an $\mathrm{NIH} \mathrm{R01} \mathrm{Al087390} \mathrm{to} \mathrm{F.D.-G,} \mathrm{and} \mathrm{a} \mathrm{K99/R00} \mathrm{Pathway} \mathrm{to} \mathrm{Independence} \mathrm{Award}$ to F.D.-G. from the National Institutes of Health 4R00MH086162-02. Work in M.B.'s laboratory was supported by ANRS and SIDACTION. N.L. is a recipient of SIDACTION fellowship.

\section{Author details}

${ }^{1}$ Department of Microbiology and Immunology, Albert Einstein College of Medicine Bronx, Bronx, NY 10461, USA. ² Laboratoire de Virologie Moléculaire, Institut de Génétique Humaine, CNRS-UPR 1142, 141 rue de la Cardonille, 34296, Montpellier cedex 5, France.

\section{Authors' contributions}

ABN performed experiments and helped with revision of the manuscript. JCV performed experiments and helped with revision of the manuscript. TEW performed experiments and helped with revision of the manuscript. NL performed, designed experiments and helped with revision of the manuscript. MB designed experiments and helped with revision of manuscript. JB helped with revision of manuscript. FDG designed experiments, wrote manuscript, and supervised the project. All authors read and approved the final manuscript.

Received: 14 February 2012 Accepted: 12 June 2012 Published: 12 June 2012

\section{References}

1. Ayinde D, Maudet C, Transy C, Margottin-Goguet F: Limelight on two HIV/ SIV accessory proteins in macrophage infection: is Vpx overshadowing Vpr? Retrovirology 2010, 7:35.

2. Hirsch VM, Sharkey ME, Brown CR, Brichacek B, Goldstein S, Wakefield J, Byrum R, Elkins WR, Hahn BH, Lifson JD, Stevenson M: Vpx is required for dissemination and pathogenesis of SIV(SM) PBj: evidence of macrophage-dependent viral amplification. Nat Med 1998, 4:1401-1408.

3. Fletcher TM 3rd, Brichacek B, Sharova N, Newman MA, Stivahtis G, Sharp PM, Emerman M, Hahn BH, Stevenson M: Nuclear import and cell cycle arrest functions of the HIV-1 Vpr protein are encoded by two separate genes in HIV-2/SIV(SM). EMBO J 1996, 15:6155-6165.

4. Gibbs JS, Lackner AA, Lang SM, Simon MA, Sehgal PK, Daniel MD, Desrosiers RC: Progression to AIDS in the absence of a gene for vpr or vpx. J Virol 1995, 69:2378-2383.

5. Belshan M, Mahnke LA, Ratner L: Conserved amino acids of the human immunodeficiency virus type $2 \mathrm{Vpx}$ nuclear localization signal are critical for nuclear targeting of the viral preintegration complex in non-dividing cells. Virology 2006, 346:118-126.

6. Jin L, Zhou Y, Ratner L: HIV type 2 Vpx interaction with Gag and incorporation into virus-like particles. AIDS Res Hum Retroviruses 2001, 17:105-111.

7. Selig L, Pages JC, Tanchou V, Preveral S, Berlioz-Torrent C, Liu LX, Erdtmann $L$, Darlix J, Benarous R, Benichou S: Interaction with the p6 domain of the gag precursor mediates incorporation into virions of Vpr and Vpx proteins from primate lentiviruses. J Virol 1999, 73:592-600.

8. Park IW, Sodroski J: Amino acid sequence requirements for the incorporation of the Vpx protein of simian immunodeficiency virus into virion particles. J Acquir Immune Defic Syndr Hum Retrovirol 1995 10:506-510.

9. Kappes JC, Parkin JS, Conway JA, Kim J, Brouillette CG, Shaw GM, Hahn BH: Intracellular transport and virion incorporation of vpx requires interaction with other virus type-specific components. Virology 1993, 193:222-233.

10. Goujon C, Riviere L, Jarrosson-Wuilleme L, Bernaud J, Rigal D, Darlix JL, Cimarelli A: SIVSM/HIV-2 Vpx proteins promote retroviral escape from a proteasome-dependent restriction pathway present in human dendritic cells. Retrovirology 2007, 4:2.

11. Srivastava S, Swanson SK, Manel N, Florens L, Washburn MP, Skowronski J: Lentiviral Vpx accessory factor targets VprBP/DCAF1 substrate adaptor for cullin 4 E3 ubiquitin ligase to enable macrophage infection. PLOS Pathog 2008, 4:e1000059. 
12. Kaushik R, Zhu X, Stranska R, Wu Y, Stevenson M: A cellular restriction dictates the permissivity of nondividing monocytes/macrophages to lentivirus and gammaretrovirus infection. Cell Host Microbe 2009, 6:68-80

13. Fujita M, Otsuka M, Miyoshi M, Khamsri B, Nomaguchi M, Adachi A: Vpx is critical for reverse transcription of the human immunodeficiency virus type 2 genome in macrophages. J Virol 2008, 82:7752-7756.

14. Bergamaschi A, Ayinde D, David A, Le Rouzic E, Morel M, Collin G, Descamps D, Damond F, Brun-Vezinet F, Nisole S, et al: The human immunodeficiency virus type 2 Vpx protein usurps the CUL4A-DDB1 DCAF1 ubiquitin ligase to overcome a postentry block in macrophage infection. J Virol 2009, 83:4854-4860.

15. Goujon C, Arfi V, Pertel T, Luban J, Lienard J, Rigal D, Darlix JL, Cimarelli A: Characterization of simian immunodeficiency virus SIVSM/human immunodeficiency virus type $2 \mathrm{Vpx}$ function in human myeloid cells. J Virol 2008, 82:12335-12345.

16. Sunseri $N$, O'Brien M, Bhardwaj N, Landau NR: Human immunodeficiency virus type 1 modified to package Simian immunodeficiency virus Vpx efficiently infects macrophages and dendritic cells. J Virol 2011, 85:6263-6274

17. Laguette N, Sobhian B, Casartelli N, Ringeard M, Chable-Bessia C, Segeral E, Yatim A, Emiliani S, Schwartz O, Benkirane M: SAMHD1 is the dendriticand myeloid-cell-specific HIV-1 restriction factor counteracted by Vpx. Nature 2011, 474:654-657.

18. Hrecka K, Hao C, Gierszewska M, Swanson SK, Kesik-Brodacka M, Srivastava S, Florens L, Washburn MP, Skowronski J: Vpx relieves inhibition of HIV-1 infection of macrophages mediated by the SAMHD1 protein. Nature 2011, 474:658-661.

19. Rice GI, Bond J, Asipu A, Brunette RL, Manfield IW, Carr IM, Fuller JC, Jackson RM, Lamb T, Briggs TA, et al: Mutations involved in Aicardi-Goutieres syndrome implicate SAMHD1 as regulator of the innate immune response. Nat Genet 2009, 41:829-832.

20. Qiao F, Bowie JU: The many faces of SAM. Sci STKE 2005, 2005:re7.

21. Zimmerman MD, Proudfoot M, Yakunin A, Minor W: Structural insight into the mechanism of substrate specificity and catalytic activity of an HD-domain phosphohydrolase: the 5'-deoxyribonucleotidase YfbR from Escherichia coli. J Mol Biol 2008, 378:215-226.

22. Lahouassa H, Daddacha W, Hofmann H, Ayinde D, Logue EC, Dragin L, Bloch N, Maudet C, Bertrand M, Gramberg T, et al: SAMHD1 restricts the replication of human immunodeficiency virus type 1 by depleting the intracellular pool of deoxynucleoside triphosphates. Nat Immunol 2012, 13:621.

23. Powell RD, Holland PJ, Hollis T, Perrino FW: Aicardi-Goutieres syndrome gene and HIV-1 restriction factor SAMHD1 is a dGTP-regulated deoxynucleotide triphosphohydrolase. J Biol Chem 2011, 286:43596-43600.

24. Goldstone DC, Ennis-Adeniran V, Hedden JJ, Groom HC, Rice Gl, Christodoulou E, Walker PA, Kelly G, Haire LF, Yap MW, et al: HIV-1 restriction factor SAMHD1 is a deoxynucleoside triphosphate triphosphohydrolase. Nature 2011, 480:379-382

25. Mahalingam S, Van Tine B, Santiago ML, Gao F, Shaw GM, Hahn BH: Functional analysis of the simian immunodeficiency virus Vpx protein: identification of packaging determinants and a novel nuclear targeting domain. J Virol 2001, 75:362-374

26. Belshan $M$, Ratner $L$ : Identification of the nuclear localization signal of human immunodeficiency virus type 2 Vpx. Virology 2003, 311:7-15.

27. Chook YM, Suel KE: Nuclear import by karyopherin-betas: recognition and inhibition. Biochim Biophys Acta 2011, 1813:1593-1606.

28. Lange A, Mills RE, Lange CJ, Stewart M, Devine SE, Corbett AH: Classical nuclear localization signals: definition, function, and interaction with importin alpha. J Biol Chem 2007, 282:5101-5105.

29. Brandariz-Nunez A, Menaya-Vargas R, Benavente J, Martinez-Costas J: IC-tagging and protein relocation to ARV muNS inclusions: a method to study protein-protein interactions in the cytoplasm or nucleus of living cells. PLOS One 2010, 5:e13785.

30. Brandariz-Nunez A, Menaya-Vargas R, Benavente J, Martinez-Costas J: Avian reovirus microNS protein forms homo-oligomeric inclusions in a microtubule-independent fashion, which involves specific regions of its C-terminal domain. J Virol 2010, 84:4289-4301.

31. Laguette N, Rahm N, Sobhian B, Chable-Bessia C, Munch J, Snoeck J, Sauter D, Switzer WM, Heneine W, Kirchhoff F, et al: Evolutionary and functional analyses of the interaction between the myeloid restriction factor SAMHD1 and the lentiviral Vpx protein. Cell Host Microbe 2012, 11:205-217.
32. Goncalves A, Karayel E, Rice Gl, Bennett KL, Crow YJ, Superti-Furga G, Burckstummer T: SAMHD1 is a nucleic-acid binding protein that is mislocalized due to aicardi-goutieres syndrome-associated mutations. Hum Mutat 2012.

33. Laguette N, Benkirane M: How SAMHD1 changes our view of viral restriction. Trends Immunol 2012, 33:26-33.

34. Sharova N, Wu Y, Zhu X, Stranska R, Kaushik R, Sharkey M, Stevenson M: Primate lentiviral Vpx commandeers DDB1 to counteract a macrophage restriction. PLOS Pathog 2008, 4:e1000057.

35. Goujon C, Jarrosson-Wuilleme L, Bernaud J, Rigal D, Darlix JL, Cimarelli A: Heterologous human immunodeficiency virus type 1 lentiviral vectors packaging a simian immunodeficiency virus-derived genome display a specific postentry transduction defect in dendritic cells. J Virol 2003, 77:9295-9304

36. Lienlaf M, Hayashi F, Di Nunzio F, Tochio N, Kigawa T, Yokoyama S, DiazGriffero F: Contribution of E3-ubiquitin ligase activity to HIV-1 restriction by TRIM5alpha(rh): structure of the RING domain of TRIM5alpha. J Virol 2011, 85:8725-8737.

37. Yee JK, Friedmann T, Burns JC: Generation of high-titer pseudotyped retroviral vectors with very broad host range. Methods Cell Biol 1994 43(Pt A):99-112.

38. Diaz-Griffero F, Perron M, McGee-Estrada K, Hanna R, Maillard PV, Trono D, Sodroski J: A human TRIM5alpha B30.2/SPRY domain mutant gains the ability to restrict and prematurely uncoat B-tropic murine leukemia virus. Virology 2008, 378:233-242

39. Scotton CJ, Martinez FO, Smelt MJ, Sironi M, Locati M, Mantovani A, Sozzani S: Transcriptional profiling reveals complex regulation of the monocyte IL-1 beta system by IL-13. J Immunol 2005, 174:834-845.

40. Kumar D, Shadrach JL, Wagers AJ, Lassar AB: Id3 is a direct transcriptional target of Pax7 in quiescent satellite cells. Mol Biol Cell 2009, 20:3170-3177.

doi:10.1186/1742-4690-9-49

Cite this article as: Brandariz-Nuñez et al.: Role of SAMHD1 nuclear localization in restriction of HIV-1 and SIVmac. Retrovirology 2012 9:49.

\section{Submit your next manuscript to BioMed Central and take full advantage of:}

- Convenient online submission

- Thorough peer review

- No space constraints or color figure charges

- Immediate publication on acceptance

- Inclusion in PubMed, CAS, Scopus and Google Scholar

- Research which is freely available for redistribution 